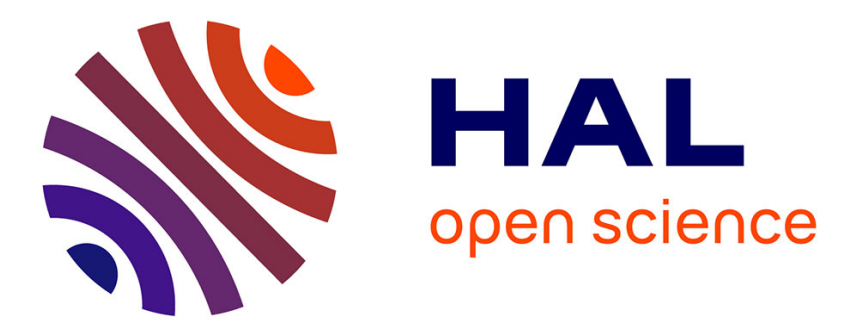

\title{
Beyond target-decoy competition: stable validation of peptide and protein identifications in mass spectrometry-based discovery proteomics
}

Yohann Couté, Christophe Bruley, Thomas Burger

\section{- To cite this version:}

Yohann Couté, Christophe Bruley, Thomas Burger. Beyond target-decoy competition: stable validation of peptide and protein identifications in mass spectrometry-based discovery proteomics. Analytical Chemistry, 2020, 10.1021/acs.analchem.0c00328 . hal-02963041

\section{HAL Id: hal-02963041 \\ https://hal.science/hal-02963041}

Submitted on 11 Oct 2021

HAL is a multi-disciplinary open access archive for the deposit and dissemination of scientific research documents, whether they are published or not. The documents may come from teaching and research institutions in France or abroad, or from public or private research centers.
L'archive ouverte pluridisciplinaire $\mathbf{H A L}$, est destinée au dépôt et à la diffusion de documents scientifiques de niveau recherche, publiés ou non, émanant des établissements d'enseignement et de recherche français ou étrangers, des laboratoires publics ou privés. 


\title{
Beyond target-decoy competition:
}

\section{stable validation of peptide and protein}

\section{identifications in mass spectrometry-based}

\section{discovery proteomics}

\author{
Yohann Couté, ${ }^{\dagger, \ddagger}$ Christophe Bruley, ${ }^{\dagger, \ddagger}$ and Thomas Burger ${ }^{*, \dagger}$ \\ †Univ. Grenoble Alpes, CNRS, CEA, INSERM, IRIG, BGE, F-38000 Grenoble, France \\ $\ddagger$ Equal contribution \\ E-mail: thomas.burger@cea.fr
}

\begin{abstract}
In bottom-up discovery proteomics, target-decoy competition (TDC) is the most popular method for false discovery rate (FDR) control. Despite unquestionable statistical foundations, this method has drawbacks, including its hitherto unknown intrinsic lack of stability vis-à-vis practical conditions of application. Although some consequences of this instability have already been empirically described, they may have been misinterpreted. This article provides evidence that TDC has become less reliable as the accuracy of modern mass spectrometers improved. We therefore propose to replace TDC by a totally different method to control the FDR at spectrum, peptide and protein levels, while benefiting from the theoretical guarantees of the Benjamini-Hochberg framework. As this method is simpler to use, faster to compute and more stable than TDC, we argue that it is better adapted to the standardization and throughput constraints of current proteomic platforms.
\end{abstract}


Most projects involving mass spectrometry (MS)-based discovery proteomics use datadependent acquisition workflows in which tandem mass (MS/MS) spectra are produced from isolated peptides. Then, peptide identification is performed by database search engines which match the experimental spectra acquired with theoretical spectra derived from a list of protein sequences ${ }^{1}$. The more the experimental spectrum resembles the theoretical spectrum, the higher the matching score. This methodology has been widely adopted, but it was soon recognized that it could lead to false positive identifications ${ }^{2}$. Indeed, among the tremendous number of spectra generated by a peptide mixture prepared from a complex biological sample, at least a few of them are expected to match an erroneous sequence, by chance. To avoid corrupting the biological conclusions of the analysis, researchers have come to rely on statistical procedures to limit the False Discovery Proportion (FDP) - i.e. the proportion of mismatches among all the peptide spectrum matches (PSMs) which look correct. As this quality control problem is ubiquitous in science, statisticians have extensively studied it. The main conclusions of these studies ( $\mathrm{See}^{3}$ for a proteomic-oriented summary) are as follows: (i) Due to the random nature of the mismatches, it is impossible to precisely compute the FDP; (ii) However, it can be estimated, as an FDR (False Discovery Rate); (iii) Depending on the experiment, the FDR will provide a more or less accurate estimate of the FDP; (iv) Therefore, practitioners should carefully select the FDR methodology, and interpret its result cautiously, making an educated guess (e.g., like a political poll before an election).

Target-decoy competition (TDC) has emerged as the most popular method to estimate the FDP in MS-based discovery proteomics ${ }^{4}$. Its success is a marker both of its conceptual simplicity and of its broad scope of application. The principle of TDC is to create artificial mismatches by searching a specific ("decoy") database of random sequences which differ from the sequences of interest (present in the "target" database) and to organize a competition between target and decoy assignments. Under the so-called Equal Chance Assumption (or ECA, stating that target mismatches and decoy matches are equally likely ${ }^{4}$ ), it is possi- 
ble, for any given cut-off score, to estimate the number of target mismatches that will be validated. Like any other estimator, TDC-FDR can lead to inconsistent estimates if the theoretical assumptions on which it is based do not hold in practice. Notably, the quality of TDC-FDR is strictly linked to the ECA validity, i.e. the decoy's capacity to adequately fool the database search engine. If it fools it too much, the TDC-FDR will overestimate the FDP; whereas if it is too unrealistic to fool the search engine, the FDP will be underestimated $^{5}$. For this reason, decoy database construction and conditions of application have been extensively studied. Results from these studies indicate that: (i) the search engine must be compliant with $\mathrm{TDC}^{6}$; (ii) In theory, the larger the decoy database, the more precise the mismatch score distribution ${ }^{7,8}$; (iii) The decoys must respect the cleavage sites ${ }^{9}$ to avoid systematic target matching regardless of spectrum quality; (iv) The influence of randomness in the construction of the decoy database can be counter-balanced by boosting strategies, leading to less volatile $\mathrm{FDRs}^{10}$; $(v)$ Decoy counting also has an influence ${ }^{8}$. In addition to these restrictions, numerous parameters have been reported and discussed to control their relative importance ${ }^{11}$. This extensive body of literature has notably contributed to installing the competition step of TDC as essential, and today, target-decoy searches without competition ${ }^{12,13}$ are scarcely ever reported. Despite the wide acceptance of TDC, a series of letters from Bret Cooper ${ }^{14,15}$ initiated a controversy regarding the observed downfall of TDC validation levels with data produced by high-resolution mass spectrometers. He provided experimental arguments to reject the idea that such downfall was simply a positive consequence of instrument evolution, leading to an increase in the numbers of peptides identified. Notably, he pointed out that very low-quality spectra incompatible with confident peptide identifications could be validated despite application of a stringent FDR cut-off. Moreover, as this phenomenon was observed with multiple widely-used search engines (Mascot, X!tandem and MS-GF+), he concluded that there was an "inherent bias" of "peptide presumption" (i.e., only peptides already listed in the target database could be identified). As this stance contradicted both empirical and theoretical evidence, a few ar- 
ticles were published arguing against this view ${ }^{16,17}$ while others confirmed ${ }^{18,19}$, maintaining the statu quo.

However, Cooper's observations can be reconciled with statistical theory. In fact, the correctness of any statistical estimate is only asymptotic: if the quality of the empirical model depicting the mismatches is improved (for instance, by increasing the size of the decoy database ${ }^{7,8}$ or by averaging a growing number of TDC-FDRs resulting from randomly generated decoy databases, in a boosting-like strategy ${ }^{10}$ ), we should end-up with a series of estimates that theoretically converges towards the FDP. Although essential, this asymptotic property is unfortunately not sufficient for practitioners, who work with a finite number of decoy databases of finite size (classically, a single decoy database of the same size as the target database). In this context, even if TDC is asymptotically unbiased (i.e., no systematic difference between the FDP and the FDR) it could sometimes lead to inaccurate FDRs (i.e., over-conservative or anti-conservative estimates) due to excessive variance (i.e., extensive stochastic fluctuations).

In this article, we shed new light on Cooper's observations, which reconcile opposing opinions: While we believe target and decoy searches can be used to accurately compute FDRs, we uphold his concerns by showing that, with state-of-the-art high-resolution instruments, the risk that the TDC strongly underestimates the FDP increases. We then describe a series of mathematical transformations of classical identification scores, to which the well-known Benjamini-Hochberg $(\mathrm{BH})$ procedure $^{20}$ and its numerous variants ${ }^{21}$ can be applied at spectrum, peptide and protein levels. This leads to an original and powerful framework that demonstrably controls the FDR without decoy databases. Altogether, the results presented demonstrate that making TDC-FDR compliant with instrument improvements requires unexpected efforts (careful implementation, fine-tuning, manual checks and computational time), whereas identification results from MS-based proteomics can be simply and accurately validated by applying alternative strategies. 


\section{Experimental Section}

\section{Sample preparation and nanoLC-MS/MS analyses}

For this work, we used the data obtained with a quality control standard composed of $E$. coli digest, analyzed according to a standard protocol.

Briefly, competent E. coli DH5 $\alpha$ cells transformed with pUC19 plasmid were grown at $37^{\circ} \mathrm{C}$ in Lysogeny broth (LB) medium containing carbenicillin before harvesting during exponential phase $\left(\mathrm{OD}_{600} \sim 0.6\right)$. After centrifugation at $3^{\prime} 000 \times g$ during $10 \mathrm{~min}$, the pellet was washed 3 times with cold Phosphate Buffered Saline (PBS) before lysis of cells using Bugbuster Protein Extraction Reagent (Novagen) containing cOmplete ${ }^{\mathrm{TM}}$, Ethylenediaminetetraacetic acid (EDTA)-free Protease Inhibitor Cocktail (Roche) and benzonase (Merck Millipore). After centrifugation at $3^{\prime} 000 \times g$ during $30 \mathrm{~min}$ and at $4^{\circ} \mathrm{C}$, the supernatant was recovered and the protein amount was measured, before protein solubilisation in Laemmli buffer.

Proteins were stacked in a single band in the top of a SDS-PAGE gel (4-12\% NuPAGE, Life Technologies) and stained with Coomassie blue R-250 before in-gel digestion using modified trypsin (Promega, sequencing grade) as described in ${ }^{22}$.

Resulting peptides were analyzed by online nanoliquid chromatography coupled to tandem MS (UltiMate 3000 and LTQ-Orbitrap Velos Pro, or UltiMate 3000 RSLCnano and Q-Exactive Plus, Thermo Scientific). The equivalent of $100 \mathrm{ng}$ of starting protein material was used for each injection. Peptides were sampled on $300 \mu \mathrm{m} \times 5 \mathrm{~mm}$ PepMap C18 precolumns (Thermo Scientific) and separated on $75 \mu \mathrm{m} \times 250 \mathrm{~mm}$ C18 columns (Reprosil-Pur 120 C18-AQ, Dr. Maisch HPLC GmBH, $3 \mu \mathrm{m}$ and $1.9 \mu \mathrm{m}$ porous spherical silica for respectively UltiMate 3000 and UltiMate 3000 RSLCnano). The nanoLC method consisted of a linear 60 -min gradient ranging from $5.1 \%$ to $41 \%$ of acetonitrile in $0.1 \%$ formic acid.

For LTQ-Orbitrap Velos Pro analyses, the spray voltage was set at $1.5 \mathrm{kV}$ and the heated capillary was adjusted to $200^{\circ} \mathrm{C}$. Survey full-scan MS spectra ( $\mathrm{m} / z$ between 400 and 1600) 
were acquired with a resolution of $60^{\prime} 000$ at $\mathrm{m} / z$ of 400 after the accumulation of $10^{6}$ ions (maximum filling time $500 \mathrm{~ms}$ ). The twenty most intense ions from the preview survey scan delivered by the Orbitrap were fragmented by collision-induced dissociation (collision energy $35 \%$ ) in the LTQ after accumulation of $10^{4}$ ions (maximum filling time $100 \mathrm{~ms}$ ). MS and MS/MS data were acquired using the software Xcalibur (Thermo Scientific). For Q-Exactive Plus analyses, the spray voltage was set at $1.5 \mathrm{kV}$ and the heated capillary was adjusted to $250^{\circ} \mathrm{C}$. Survey full-scan MS spectra $(\mathrm{m} / \mathrm{z}$ between 400 and 1600$)$ were acquired with a resolution of $60^{\prime} 000$ at $m / z$ of 400 after the accumulation of $10^{6}$ ions (maximum filling time $200 \mathrm{~ms}$ ). The ten most intense ions were fragmented by higher-energy collisional dissociation (normalized collision energy 30\%) after accumulation of $10^{5}$ ions (maximum filling time 50 ms) and spectra were acquired with a resolution of $15^{\prime} 000$ at $m / z$ of 400 . MS and MS/MS data were acquired using the software Xcalibur (Thermo Scientific).

\section{MS data analysis}

Data were processed automatically using Mascot Distiller software (version 2.6, Matrix Science). Peptides and proteins were identified using Mascot (version 2.6) through concomitant searches against Escherichia coli K12 reference proteome (20180727 version downloaded from UniProt), and/or custom made decoy databases (reversed or shuffled sequences - see below). Trypsin/P was chosen as the enzyme and 2 missed cleavages were allowed. Precursor and fragment mass error tolerance has been variably adjusted as described in the manuscript. Peptide modifications allowed during the search were: carbamidomethylation (C, fixed), acetyl (Protein N-ter, variable) and oxidation (M, variable). Proline software ${ }^{23}$ was used to filter the results: conservation of rank 1 peptide-spectrum match (PSM) and single PSM per query. 1\% FDR control was performed with various methods, as described in the Results section. Precisions regarding the choice of the score (individualized vs. contextualized) as well as of alternative search engines can be found in Supporting Information S2.3 and S4.1. 


\section{Decoy database generation}

For classical TDC experiments (Figures 1 and 2), we used the following procedure: The target database was reversed by using the Perl script (decoy.pl) supplied with Mascot software and the generated decoy database was appended to the target one before concatenated search. From our observations, slightly different procedures (shuffled vs. reversed, accounting for tryptic cleavage site, etc.) yields similar results, which concurs with prior knowledge ${ }^{11}$.

To compute the Empirical Null (EN) FDRs ${ }^{24}$ shown in Figure 3, we relied on the model provided by a shuffled database not used in competition with the target database ${ }^{12,13}$ combined with a boosting strategy ${ }^{10}$ ( i.e. a procedure averaging the FDR estimates from multiple

shuffled decoy databases ). For this study, we used 10 shuffled databases, each with a length equal to that of the target database, and produced by a shuffling procedure which respected cleavage sites to maintain the precursor mass distribution ${ }^{9}$ ( $\mathrm{R}$ code available in Supporting Information S1.1).

\section{Results and Discussion}

\section{TDC and Benjamini-Hochberg procedures yield different FDRs}

To counteract the the drop-off in validation levels observed with modern MS instruments ${ }^{14,15}$, we suspected that the Benjamini-Hochberg $(\mathrm{BH})$ approach to FDR control $^{20}$ could be an interesting alternative. Discrepancies between BH and TDC have already been reported ${ }^{25,26}$, but unfortunately direct quantitative comparisons in terms of bias (i.e., systematic error) and variance (i.e., lack of stability) are impossible. ${ }^{27}$ Such comparisons would require the FDP to be precisely known, although it is not accessible, even when using a controlled dataset. Thus, to better grasp the possible differences in behavior between FDRs estimated by the TDC and BH approaches, we reproduced Cooper's experiment with the following extensions: First, both FDRs were computed simultaneously on the same datasets; Second, 
the settings for many experimental parameters were varied: we used multiple analytical replicates, different instruments, as well as various combinations of precursor and fragment mass tolerance sets.

To compute a BH-FDR, the search engine must provide PSM scores which can be related to p-values. Fortunately, numerous state-of-the-art search engines do $\mathrm{so}^{28}$ : For instance, Mascot provides scores in the form

$$
S=-10 \cdot \log _{10}(p) \quad \text { or } \quad p=10^{-\frac{S}{10}}
$$

where $p$ is a p-value. Andromeda provides a similar calculation, although the score is not directly accessible as it is only an intermediate computation (see Supporting Information S4.1). PepProbe, InsPecT and MyriMatch directly provide p-values as scores, and SEQUEST scores can be transformed into p-values through the application of dedicated wrappers e.g. ${ }^{25,26}$. In theory, p-values always distribute uniformly across the $[0,1]$ interval $^{3}$. However, when they do not in practice, they are termed ill-calibrated or miscalibrated. As miscalibration can affect $\mathrm{BH}$ estimates, potentially making them incorrect, tools have been developed to check or improve the p-value calibration (see Supporting Information S1.2).

We applied both TDC and BH methodologies to results acquired with a Q-Exactive Plus instrument on ten analytical replicates of an E. coli lysate. MS and MS/MS spectra were acquired at relatively high resolutions (70,000 and 17,500 at $\mathrm{m} / \mathrm{z}$ of 200 , respectively). We used Mascot to run a TDC search (see Experimental Section) and we considered four combinations of mass tolerance tuning at the precursor and fragment levels: LL, HL, LH and $\mathrm{HH}$ (where L stands for low precision or large tolerance, and $\mathrm{H}$ for high precision or narrow tolerance), the final combination $(\mathrm{HH})$ corresponds to the tolerance levels generally used on our platform for Q-Exactive data analysis. In parallel, we ran target-only searches using the same parameters. Scores were then converted into p-values using Eq. 1, and the calibration of the resulting p-values was assessed as reported in Supporting Information S2.1. Finally, 

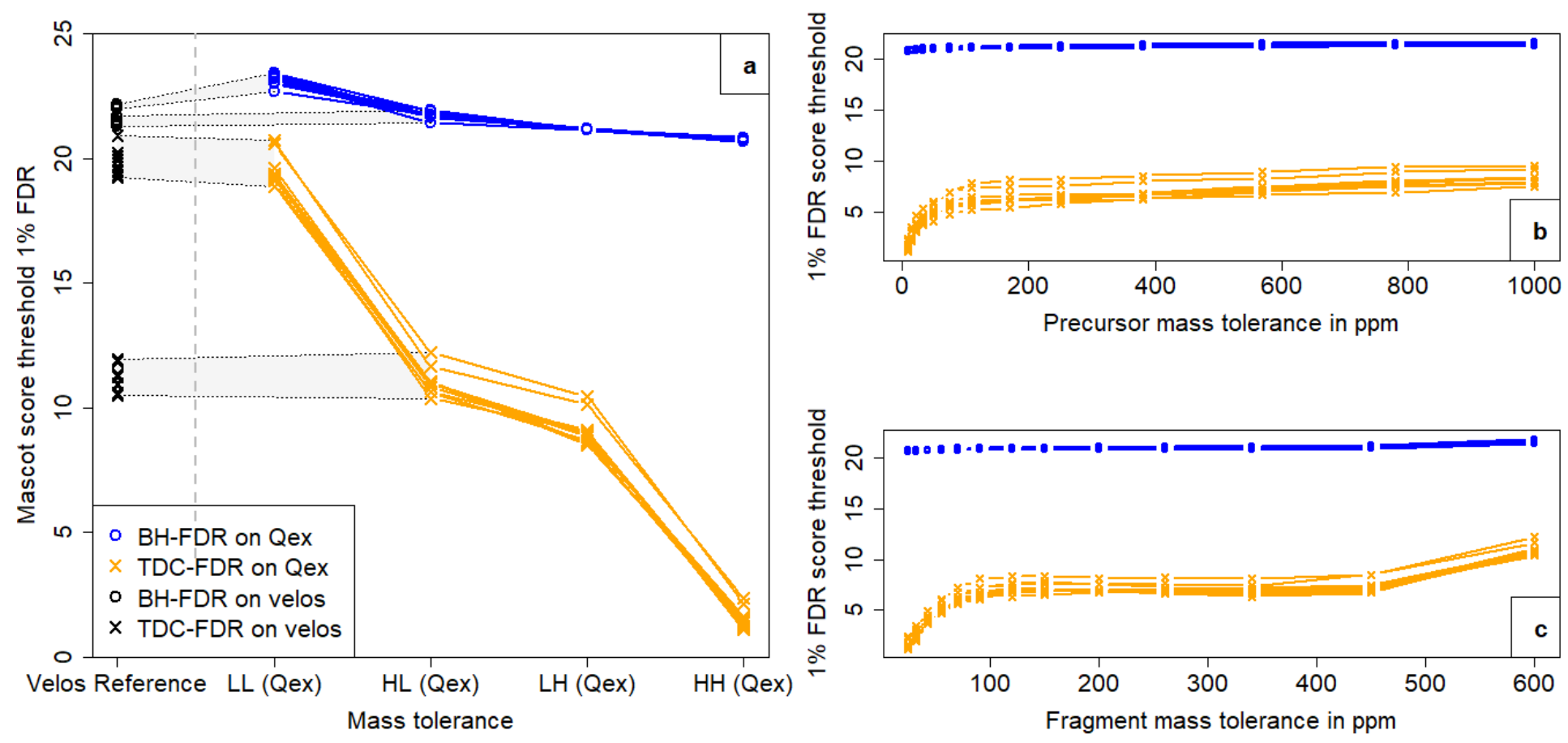

Figure 1: Score thresholds obtained when applying TDC (Orange) and BH (Blue) filtering at an FDR of 1\%, as a function of the search engine mass tolerance parameters, for 10 samples analyzed with a Q-Exactive Plus (Qex) instrument. (a) Precursor and fragment mass tolerances were tuned to the LL, LH, HL and HH settings: LL assumes the MS and MS/MS data were acquired at low resolutions for the precursor and fragment masses $(1 \mathrm{Da}$ and 0.6 Da, respectively); HL uses mass tolerances of $10 \mathrm{ppm}$ and $0.6 \mathrm{Da}$, respectively; LH uses mass tolerances of $1 \mathrm{Da}$ and $25 \mathrm{mmu}$; and finally, $\mathrm{HH}$ uses mass tolerances of $10 \mathrm{ppm}$ and $25 \mathrm{mmu}$ (which corresponds to classical parameters for database searches performed with Qex data). The black lines encompass thresholds resulting from similar analyses performed on an LTQ-Orbitrap Velos Pro (Velos) with LL and HL settings. (b) Refined analysis of the FDR threshold's sensitivity to precursor mass tolerance tuning (Qex data, fragment tolerance $=25 \mathrm{mmu}$ ). (c) Refined analysis of the FDR threshold's sensitivity to fragment mass tolerance tuning (Qex data, precursor tolerance $=10 \mathrm{ppm}$ ). 
the classical BH step-up procedure for p-value adjustment was applied (see Data and code availability).

Figure $1 \mathrm{~A}$ shows the score thresholds obtained at $1 \% \mathrm{FDR}$ as a function of the mass tolerance combinations applied (see Supporting Information S2.2 for numerical values). At first glance, the drop-off in validation scores is obvious with TDC, whereas it is almost immaterial with BH. To avoid drawing sweeping conclusions on this impression, we performed complementary experiments. First, to better capture the influence of mass tolerance tuning, starting from the $\mathrm{HH}$ setting, we progressively extended the mass tolerance range, either for the precursor (Figure 1B) or for fragment masses (Figure 1C). The trends observed in these analyses support the one shown in Figure 1A. Moreover, they confirm that tolerances at precursor and fragment levels have a greater influence on threshold scores determined using TDC compared to BH.

Second, to confirm that the results obtained by reducing the mass tolerance in the search parameters mimics results obtained with lower-resolution instruments, we analyzed another batch of ten analytical replicates of the E. coli lysate submitted to MS/MS on a LTQOrbitrap Velos Pro with mixed resolutions: high resolution in MS mode (Orbitrap analysis) and lower resolution in MS/MS mode (linear ion trap analysis). Database searches were performed using the HL and LL tuning paramaters, and FDR thresholds were computed as above. Interestingly, the matching between the results from Q-Exactive and LTQ-Orbitrap data obtained using the same thresholds was excellent. This result justifies our methodology: from an FDR viewpoint, switching to analysis of a lower-resolution dataset using an appropriately-tuned search engine, or retaining the higher-resolution data while substantially increasing the mass tolerances, produces roughly equivalent outputs.

Therefore, we can interpret the different database search sets as surrogates for the recent improvements to instrumental capabilities: when TDC was first applied to data from lowresolution instruments, $\mathrm{TDC}$ and $\mathrm{BH}$ produced roughly similar results in terms of score cut-off to reach 1\% FDR. Since then, the resolution of MS instruments has progressively 
increased, and now TDC and BH diverge considerably when applied to the same lists of putative PSMs, so that at least one of them (but possibly both) yields an incorrect FDR control.

\section{$\mathrm{BH}$ is more stable than TDC}

Based on the results presented in Figure 1, BH appears more stable than TDC, both within and between experimental settings: First, for each tuning taken individually, the TDC threshold was less stable than its BH counterpart, as the set of ten cut-off scores was more dispersed with TDC. Second, depending on the mass tolerances applied when performing database searches, the TDC threshold on the Mascot score varied from 1.11 to 20.73 ( with HH and LL settings, respectively), whereas its BH counterpart was more stable (between 20.67 and 23.43).

A part of the TDC's instability (relative to $\mathrm{BH}$ ) can be explained by the random nature of decoy sequence generation ${ }^{10}$, regardless of the search engine used. However, at first glance, there is no reason to assume that the remaining reported instability (notably the drop in score) is: (1) specifically linked to the TDC; (2) not a (much more important) bias issue. Consequently to point (1), it could make sense to question the algorithmic specificities of the search engine (here Mascot), as Cooper first $\operatorname{did}^{14}$. Unfortunately, he then reported ${ }^{15}$ similar pitfalls (at least for the precursor tolerance parameter) with X!tandem and MS-GF+ (formerly known as MS-GFDB). In addition, we discovered similar effects with Andromeda (Maxquant environment, see Supporting Information S2.3). Finally, our experiments demonstrate that not only the precursor mass tolerance set, but also, the fragment mass tolerance defined contribute to this effect. As for point (2), we hereafter demonstrate that even if the dependence on the search parameter is assumed to be a stability issue only (instead of a bias issue), it should nonetheless lead to legitimacy question TDC use. 


\section{TDC instability can lead to anti-conservative FDRs}

Naturally, more stable FDRs should be preferred, however less stable ones are not necessarily untrustworthy. As differently parametrized searches yield distinct putative PSM lists, different (correct) FDRs would make sense. However, the gap between BH and TDC cut-offs in the HH setting is alarming, as the PSMs identified in the target database before validation were the same with both methods. The very low cut-off scores obtained with TDC while using the HH setting led us to question the TDC procedure: Even if TDC is assumed to be unbiased, can its instability result in FDRs that are sometimes conservative (e.g. LL cases) and sometimes not (e.g. HH cases)? This context-dependent anti-conservativeness would clearly make use of the TDC approach less than reliable, as false discoveries would no longer be controlled.

To answer this question, we relied on the following rationale for FDR control: When a PSM list is filtered at $1 \%$ FDR, it does not mean that we accept $1 \%$ of additional poor matches in the result. On the contrary, it means that, even though all the validated PSMs

apparently correspond to matches of sufficient quality, $1 \%$ of them are spurious (randomly distributed over the full range of scores, not necessarily matches with the lowest scores). Although it appears counter-intuitive, this property has already been empirically confirmed ${ }^{29}$. Therefore, using only the HH setting and examining the list of PSMs with low scores validated by TDC is insightful to assess the conservativeness of this approach. We concretely did so with the results from the first analytical replicate, where 12209 PSMs were validated after applying TDC, leading to an expected number of mismatches at 1\% FDR equal to 122. From the validated list, we therefore randomly selected 150 PSMs with a Mascot score $<10$. The quality of the matches between theoretical and experimental spectra was obviously too low to yield confident identifications in the vast majority of cases (see Supporting Information S2.4). Based on this sample, the $[1.61 ; 10[$ interval (which corresponds to only 635 PSMs, i.e. $5.53 \%$ of 12209) already contains more mismatches than the number expected for the entire dataset. This result is incompatible with $1 \%$ FDR validation, as random misiden- 
tification depicting apparently correct matches can also be expected at higher scores ${ }^{29}$. In other words, on this dataset using the HH setting, the TDC FDR did not conservatively estimate the FDP.

These observations confirm those reported by Cooper. Although they are not sufficient to conclude on an intrinsic bias of TDC, they do confidently show that the drop-off in of validation cut-off scores is associated with, at least, an increased risk of anti-conservative FDR.

\section{Mechanistic explanation for TDC's downfall}

The above results contrast with the theoretical guarantees that have been published for $\mathrm{TDC}^{7,30,31}$. However, none of them account for the application of preliminary filters which reduce the number of decoy competitors, while Cooper's controversy is rooted in such filters. This distinction may explain the discrepancy: Depending on the instrument's accuracy when measuring the precursor mass, due to variable resolving powers, and assuming the search engine is tuned accordingly, a larger or smaller number of decoys are considered possible competitors for a given spectrum. Thus, MS data acquired with high-resolution mass spectrometers and analyzed using search engines in which narrow mass tolerances are de facto set leads to smaller numbers of decoy matches. Therefore, the ECA may no longer apply and the FDR may be underestimated. This situation can be experimentally observed while looking at the distributions of the PSMs identified in the decoy database using the LL and $\mathrm{HH}$ settings. Indeed, the number of decoy identifications, as well as their corresponding scores, were strongly decreased in the HH setting compared to the LL one (see Figure 2), and the very low number of decoy PSMs with the HH setting cannot provide an accurate FDR estimation.

As TDC appears to be inaccurate when the number of decoy challengers is too small, one straightforward solution would be to enlarge the database in proportion. Although the link between mass tolerance and decoy database size can be efficiently exploited to limit the 

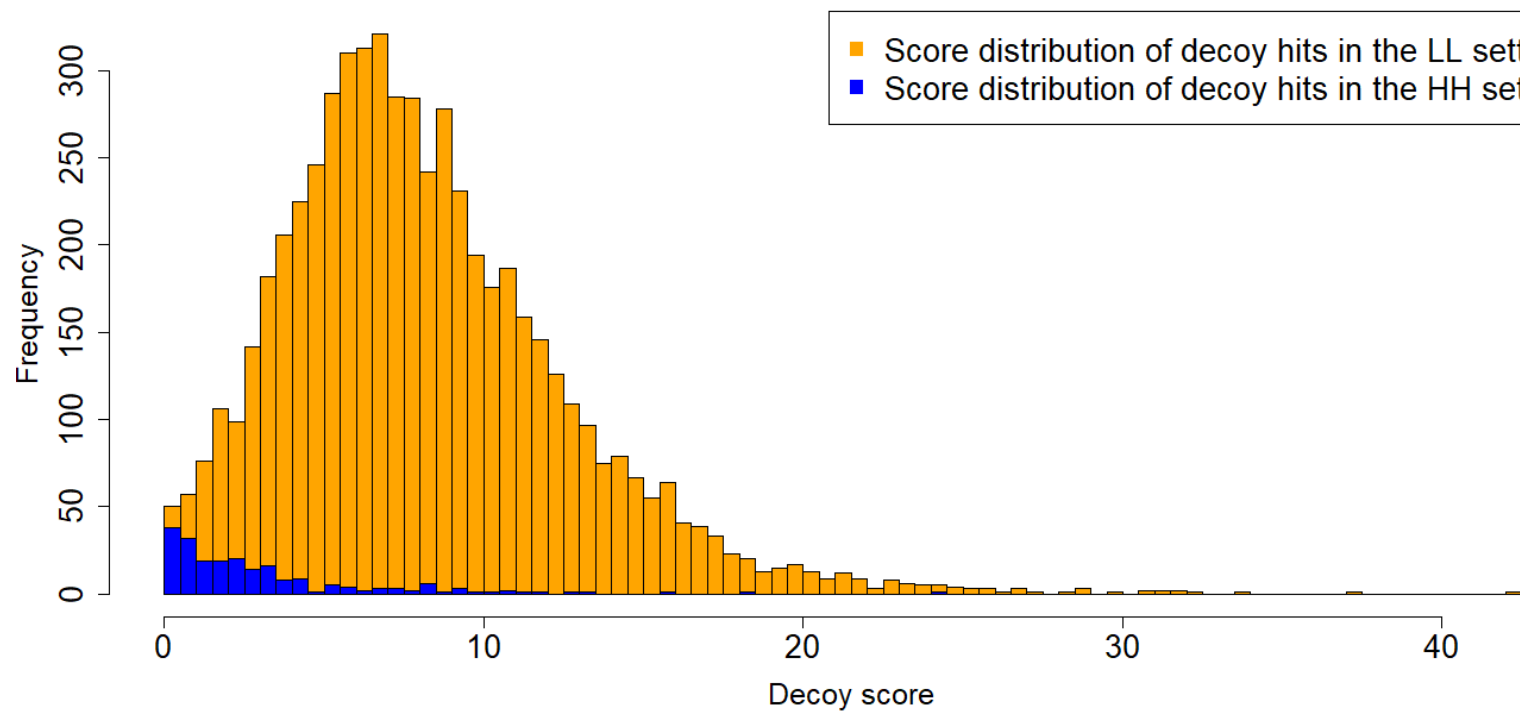

- Score distribution of decoy hits in the LL setting

- Score distribution of decoy hits in the $\mathrm{HH}$ setting

Figure 2: Distributions of scores for PSMs identified in the decoy database using the LL (orange) and HH (blue) settings. Data correspond to replicate 1, Qex analysis. After concomitant searches in target and decoy databases using LL and HH parameters, the scores for the PSMs identified only in the decoy database, and without FDR filtering, were collected and represented as histograms (x-axis: score, bin 0.5; y-axis: frequency).

computational cost of repeated searches ${ }^{32}$, it was, unfortunately, inefficient in our case (see Supporting Information S4.2).

\section{Decoy-based empirical null estimation and BH FDRs are consistent}

An alternative means to investigate the above mechanistic intuition is to build a strategy in which the quality of decoy matches is preserved, regardless of the stringency of the database's preliminary search filters; and then to examine how it estimates the FDR. The statistical theory of empirical null estimation ${ }^{24}$ is based on making false discoveries look like true ones. Even though this theory has already been applied in various proteomics contexts (giving rise to entrapment methods ${ }^{27,33-37}$ ), it provided us with a nice framework to elaborate on. Surprisingly, we found that a fair (but nevertheless unstable) empirical estimate of the null distribution could be obtained by performing a separate decoy search, without competition ${ }^{12,13}$. To address the lack of stability, we averaged 10 repeated estimations, each 

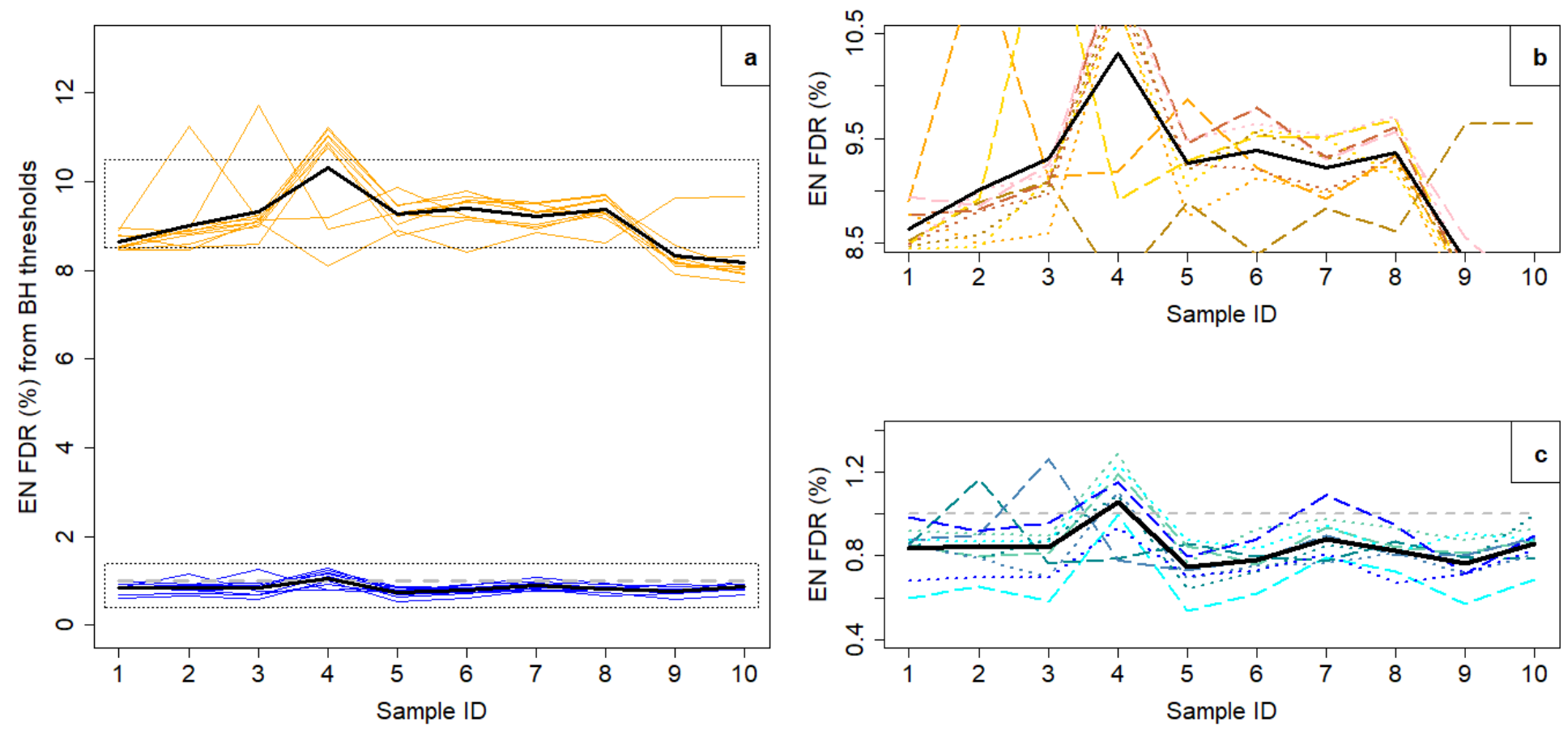

Figure 3: (a) Empirical Null (EN) FDRs computed for each replicate matched against 10 randomly generated (shuffled) decoy databases, according to its $1 \%$ FDR validation score threshold (gray dashed line), computed by applying BH (blue) and TDC (orange) methodologies; The black continuous lines show the average of the 10 EN FDRs (boosted estimate). (b) and (c) Zooms of the two framed areas in (a), with different shades of blue (resp. orange) and of line types (dot or dash) for better shuffle discrimination.

based on a different shuffled database ${ }^{10}$ (see Experimental Section). For this reason, for each of the 10 sample replicates of E. coli lysate analysed with the Q-Exactive Plus, Figure 3 shows the 10 empirical null (EN) FDRs corresponding to the cut-off scores obtained using BH and TDC 1\% FDR filtering (HH setting).

The difference is striking: although BH thresholds (Mascot scores between 20.67 and 20.84) produced EN FDRs slightly below $1 \%$ (between $0.53 \%$ and $1.29 \%$, with an average $\approx 0.84 \%$ ), those obtained with TDC (scores between 1.11 and 2.37) led to 10-fold larger EN FDRs (between $7.72 \%$ and $11.71 \%$, with an average $\approx 9.1 \%$ ). This result is insightful for three reasons: (i) The fact that two orthogonal methods to compute an FDR (namely $\mathrm{BH}$ and EN) provided concurring results is an evidence supporting their correctness; (ii) it 
confirms that TDC can lead to considerable FDR under-estimations if used inappropriately; (iii) it shows that in contrast to Cooper's concerns, the concept of "peptide presumption" is not inherently biased, since by applying an appropriate decoy search strategy, it is possible to cope for the cut-off downfall and to recover coherent FDRs.

\section{Practical comparison of EN and $\mathrm{BH}$ approaches}

To summarize, the EN approach implemented here essentially amounts to averaging multiple target-decoy searches without competition, and can be viewed as an improvement of a 12years-old method ${ }^{12,13}$ well-fitted in the proteomics landscape, yet outmoded. In contrast, the $\mathrm{BH}$ approach is mainly theoretically motivated, and even though it is double the age, it is scarcely used in proteomics. As both methods provide concurring estimates, the one to promote mainly depends on their respective conditions of applicability and ease of use.

For BH: If we compare the EN FDRs derived from the BH-thresholds on Figure 3 with the expectation (i.e. 1\%), BH appears to provide a slight overestimation. This result is probably due to the previously described over-conservative property of the $\mathrm{BH}$ estimator ${ }^{38}$. Moreover, as previously mentioned, $\mathrm{BH}$ requires that the p-values first be checked for calibration. Fortunately, many methods can be used to limit BH over-conservativeness, and applying the best one can be done concomitantly with the calibration assessment, by means of a simple visual tool ${ }^{21}$ (see CP4P description in Supporting Information S1.2). Finally, the BH FDR is extremely rapidly computed and does not require any decoy database.

For EN: Figure 3 shows a high dependency on the different decoy databases: From one randomly generated version to another, the FDR estimated varies significantly. At first glance, FDRs around $1 \%$ seem slightly more stable than those around $10 \%$. However, after normalization relative to the mean FDR value, it is actually the opposite that occurs (mean coefficient of variation of $13.90 \%$ around $1 \%$ EN FDR, versus $5.82 \%$ around $10 \%$ EN FDR). This observation can easily be explained: With lower FDR thresholds, fewer decoys passed the threshold, and as a result, the statistics were computed on smaller sample sizes, inherently 
more sensitive to randomization. This explains why it is necessary to average multiple FDRs with different randomly generated decoy searches, despite the additional computational and practical complexity. However, depending on the complexity of the experiment, the precise number of searches required to stabilize the FDR cannot be estimated and will require manual trials. Moreover, if the experimental design requires iterative filtering of the database search (e.g. multiple-pass identifications ${ }^{39}$ ), it is possible that the same anti-conservativeness issue will arise as with the competition step, so that additional caution should be applied. Therefore, compared to the EN strategy, the BH procedure is appealing for its simplicity and stability.

\section{FDR control at peptide level using the $\mathrm{BH}$ procedure}

The difficulty of inferring peptide- and protein-level knowledge from spectrum-level information, while applying quality control criteria, has been widely addressed in the literature ${ }^{40,41}$. However, to our knowledge, all available inference systems require a preliminary decoy search to propose a peptide- or protein-level FDR. Today, combining multiple levels of FDR control has become accepted standard good practice. We therefore propose a generic procedure to extend the BH-FDR approach to peptide and protein levels. Moreover, the proposed method is independent of the chosen inference rules (see Supporting Information S3.1). Hereafter, we assume that the inference rules selected unambiguously define which PSMs should be used in peptide scoring, as well as which peptides contribute to protein group scoring ${ }^{36,37,42}$, and we focus on the scoring methods applied.

The most classical peptide scoring methods assume that each peptide is identified by the spectrum with the highest PSM score amongst the $Q$ matching spectra ${ }^{35-37,43}$. In this setting, it makes sense to define the peptide score as equal to the best PSM score ${ }^{35}$. Formally, if the PSM score between peptide sequence $\mathbf{s e q}_{i}$ and spectrum $q$ is referred to as $S_{i q}$, then, the

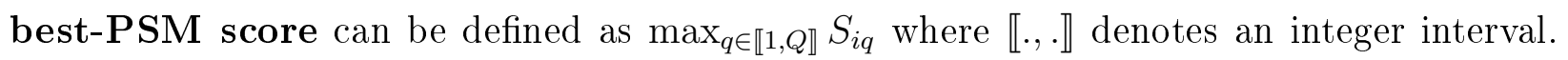
This score can potentially be used to compute a TDC-FDR, but not a BH-FDR. Indeed, 
its probabilistic counterpart cannot be well-calibrated (the minimum of several calibrated p-values is non-uniformly distributed, see Figure S1). Fortunately, according to the following proposition, it is possible to modify the best-PSM score by applying a formula akin to Šidák correction $^{44}$ and thus to recover correct calibration:

Proposition 1 Let $S_{1}, \ldots, S_{n}$ be a set of $n$ scores of the form $S_{\ell}=-10 \log _{10}\left(p_{\ell}\right),(\ell \in \llbracket 1, n \rrbracket)$ where $p_{\ell}$ is realizations of $n$ i.i.d. $\mathbb{R}_{+}$random variables, $X_{1}, \ldots, X_{n}$. If $X_{\ell} \sim \mathcal{U}[0,1] \forall \ell$, then,

$$
Y=1-\left(1-10^{-\frac{\max _{\ell}\left(S_{\ell}\right)}{10}}\right)^{n}
$$

uniformly distributes over the range $[0,1]$.

Proof: See Supporting Information S3.2.

Therefore, (See Supporting Information S3.2 and S3.3 for the full derivations), the peptide p-value $p_{i}^{\diamond}$ and peptide score $S_{i}^{\diamond}$ of peptide sequence $\mathbf{s e q}_{i}$ can be defined as:

$$
p_{i}^{\diamond}=1-\left(1-10^{-\frac{\max q\left(S_{i q}\right)}{10}}\right)^{Q} \quad \text { and } \quad S_{i}^{\diamond}=-10 \cdot \log _{10}\left(p_{i}^{\diamond}\right)
$$

\section{FDR control at protein level using the $\mathrm{BH}$ procedure}

To define protein-level scores and p-values, fragment matches for PSM scores were considered equivalent to what peptide matches are for protein scores. This equivalence led us to rely on Fisher's test to define protein scores/p-values from the scores of the best subset of peptides. Similar approaches have frequently been investigated in the literature ${ }^{32,36,37,42}$ and the full derivation is presented in Supporting Information S3.4. To the best of our knowledge, we are the first to discuss the adaptation of Fisher's methodology from its original context (meta-analysis) to proteomics by explicitly considering $(i)$ risks of anti-conservativeness due to dependent peptides (see Supporting Information S3.5); (ii) the impact of the poorly conclusive peptide-level evidence in an open-world assumption context (see Supporting In- 
formation S3.6). Finally, for a protein sequence $\mathbf{s e q}_{\pi}$ identified by $K$ specific peptides with scores $S_{1}^{\diamond}, \ldots, S_{K}^{\diamond}$, the protein p-value is defined as:

$$
p_{\pi}^{\star}=\min _{A \in 2\{1, \ldots, K\}}\left[\int_{0.2 \ln (10) \cdot \sum_{k \in A} S_{k}^{\odot}}^{\infty} f_{2 \cdot|A|}(x) d x\right]
$$

and the protein score as $S_{\pi}^{\star}=-10 \log _{10}\left(p_{\pi}^{\star}\right)$, where: $2^{\{1, \ldots, K\}}$ is the powerset of the set of $K$ peptides identified; $A$ is a peptide set with cardinality $|A| \leq K$; and $f_{2 \cdot|A|}$ is the density function of the $\chi^{2}$ distribution with $2 \cdot|A|$ degrees of freedom. Although possibly obscure at first glance, $p_{\pi}^{\star}$ simply interprets as the p-value resulting from Fisher combined probability test applied to the subset of peptides which best explains the protein (see Supporting Information S3.4).

\section{Experimental assessment of BH FDR at peptide and protein levels}

The ten replicate analyses of $E$. coli lysate were validated at $1 \%$ FDR by applying the BH procedure to the PSM, peptide and protein scores. To do so, only a target database search was necessary. However, and because it delivered a striking illustration of the capacity of the proposed framework to distinguish false identifications, we introduced shuffled sequences in the searched database to assess the results (see Experimental Section). We considered a challenging scenario where the number of decoys was set to five times the number of target sequences. Table 1 summarizes the average (across the 10 replicates) cut-off scores as well as the average counts for validated PSMs, peptides and proteins in both target and fivefold shuffled databases (see Table S2). Although the corresponding proportions must not be interpreted as FDRs, it is interesting to discuss them: First, despite the fivefold decoy over-representation, each of the three validation levels (PSM, peptide or protein) taken individually was sufficient to provide a decoy ratio below the FDP expectation of $1 \%$ at any level. Second, the three validation strategies provided broadly concurring filters and validated protein list sizes. Third, some discrepancies between the three validation strategies 
exist (for instance, when filtering at protein level, PSMs with low score are validated because they belong to proteins which are confirmed by other high scoring peptides), leaving room to refine validation with appropriate multi-level filters, as discussed below.

Table 1: Average (across the $10 \mathrm{E}$. coli replicates) minimum score and PSM, peptide and protein counts assigned as target and decoy in the raw dataset (No validation), as well as after validation by one of the three following rules: $1 \%$ BH-FDR at PSM level (PSM validation), $1 \%$ BH-FDR at peptide level (Peptide validation) and 1\% BH-FDR at protein level (Protein validation).

\begin{tabular}{||c|c|c|c||c|c|c||c|c|c|c||c||c|}
\multicolumn{1}{l||}{} & \multicolumn{3}{c||}{ No validation } & \multicolumn{3}{c||}{ PSM validation } & \multicolumn{3}{c||}{ Peptide validation } & \multicolumn{3}{c|}{ Protein validation } \\
& PSM & Pep. & Prot. & PSMs & Pep. & Prot. & PSM & Pep. & Prot. & PSM & Pep. & Prot. \\
\hline Min score & 0.002 & 0.002 & 0.002 & 21 & 19.676 & 21.187 & 0.023 & 20.963 & 21.163 & 0.013 & 0.021 & 22.534 \\
\hline \#Targets & 12020.2 & 9351.3 & 1466.5 & 10233.6 & 8180.3 & 1297.7 & 10736.2 & 8159.5 & 1297.9 & 11836.6 & 9169.4 & 1294.6 \\
\hline \#Decoys & 873.3 & 817.2 & 786 & 11.5 & 10.9 & 10.7 & 11.1 & 10.2 & 10 & 13.8 & 12 & 9.2 \\
\hline
\end{tabular}

\section{Conclusions}

This work sheds new light on a crucial step in bottom-up proteomics experiments: the validation of identification results. First, it illustrates that the TDC and BH estimates of the FDP have progressively diverged as MS accuracy has improved. Our results demonstrated that this divergence originated in the TDC's lack of stability with respect to the precursor and fragment mass tolerances set during database searches. Although this lack of stability can be partially counteracted by suppressing the competition step of $\mathrm{TDC}^{12,13}$, the instability induced by the random generation of decoy sequences remains ${ }^{10}$. Therefore, even though target-decoy strategies can be refined to partially cope with this instability, the results are not satisfactory as these strategies: (i) are not as stable as BH, in particular at lower FDRs; (ii) are more complex to organize (implementation, computational cost) and require additional manual checks; (iii) do not provide any guarantee of reliable FDR estimates in the future (on datasets acquired with even higher resolution next generation instruments for which narrower mass tolerances can be expected; or with pipeline modifications that change the number of target and decoy candidates).

Second, this work provides new peptide and protein scores which demonstrably respect 
the calibration conditions of the BH procedure. Indeed, implementing BH-FDR at PSM-, peptide- and protein-level is straightforward (see Data \& code availability) and its practical use within a preexisting platform pipeline requires no precise tuning. Moreover, our results highlighted that, despite slightly different behavior, any of these scores alone is sufficient to conservatively validate a proteomics dataset at PSM, peptide and protein levels. This finding suggests that various strategies could be developed to comply with different objectives: If the expected output is a protein list, then it is probably most appropriate to control the FDR at protein-level. However, in studies seeking to refine discrimination between proteoforms sharing many subsequences, it may be more relevant to validate at peptide level. Finally, when quantifying proteins, extracting the ion current for misidentified spectra produces erroneous results, making validation at PSM level necessary. Beyond these considerations, acting at different levels of filtering may also improve the quality of the validated identifications, although this assertion requires further investigation. For example, multiple FDRs are classically used sequentially, following the inference process (starting at PSM level and ending at protein level); using a reverse order or parallel filtering may also be of interest to preserve the calibration necessary to the $\mathrm{BH}$ procedure.

Based on these results, we propose an overhaul of how FDR is estimated in discovery proteomics using database searching and suggest replacing TDC by BH-FDR. Nevertheless, as a theoretical research field, TDC remains of interest. The original idea proposed by Elias and Gygi ${ }^{4}$ has stimulated the field of theoretical biostatistics and led to the idea that simulating null tests from the data (termed knockoffs instead of decoys) could produce efficient alternatives to $\mathrm{BH}$ procedures, which demonstrably control the FDR ${ }^{30,31,45}$. Transferring these theoretical results into biostatistics routines that can be applied on a daily basis still requires some investigation ${ }^{46,47}$. However, they will hopefully contribute to computational proteomics in the future, as an example of an interdisciplinary virtuous circle. 


\section{Data and code availability}

Implementing BH-FDR at PSM-, peptide- and protein-level is straightforward. First, if the scores of all the PSMs indicating a given peptide sequence are stored as a vector, psm.scores, then, the peptide p-value pep.pval and peptide score pep.score can be determined by applying the following $\mathrm{R}$ code:

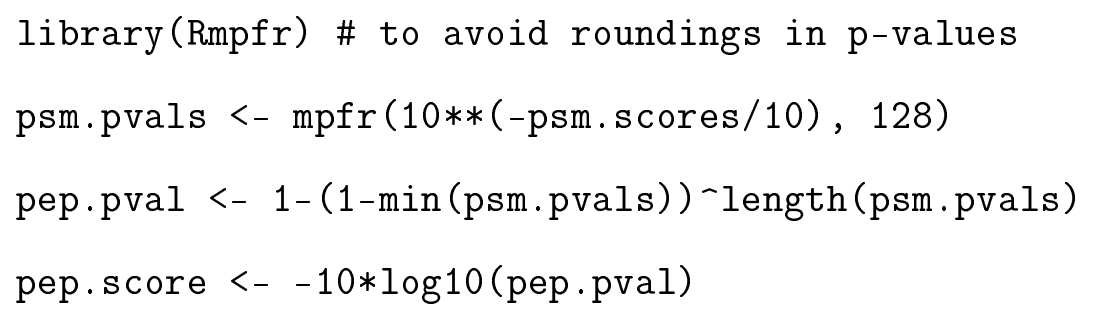

Then, the protein score prot.score and p-value prot.pval for a protein for which peptide scores are stored in a vector pep.scores can be computed using the following code:

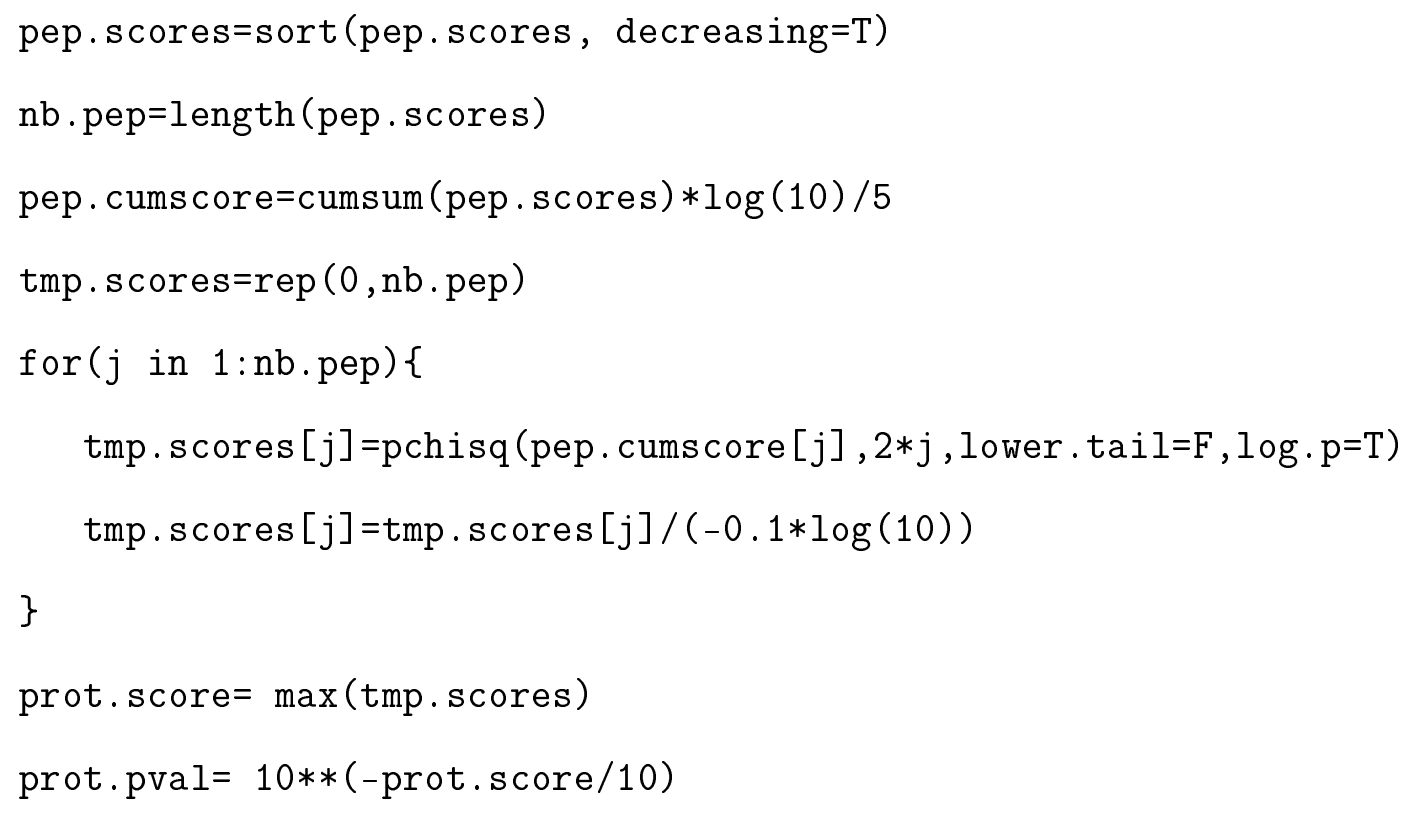

Once the peptide scores and protein scores are available alongside the PSM scores provided by the search engine, the BH procedure can simply be run by applying the p.adjust () $\mathrm{R}$ function (base function). All these scores (and the $\mathrm{BH}$ procedure) are also implemented in Proline software ${ }^{23}$, written in Java/Scala, so that any proteomics data analyst can use 
them whatever their coding skills. The mass spectrometry proteomics data have been deposited to the ProteomeXchange Consortium via the $\mathrm{PRIDE}^{48}$ partner repository with the dataset identifier PXD016669 and 10.6019/PXD016669 (https://www.ebi.ac.uk/pride/, Username: reviewer43825@ebi.ac.uk, Password: eA7lMPa7). A portable version of Proline software can be downloaded at ftp://ftp.cea.fr/pub/edyp/Proline/BH-FDR/. For each of the ten $E$. Coli replicates, it contains the search results from Mascot after the database search in the target and the fivefold decoy databases.

\section{Acknowledgments}

This work was supported by grants from the French National Research Agency: ProFI project (ANR-10- INBS-08), GRAL project (ANR-10-LABX-49-01), Data@UGA and SYMER projects (ANR-15-IDEX-02) as well as MIAI @ Grenoble 718 Alpes (ANR-19-P3IA-0003). The authors are grateful to EDyP platform engineers, who performed the various quality control analyses used in this work, and notably to Alexandra Kraut for the sample preparation, as well as the ProFI developers of Proline software (http://www.profiproteomics.fr/ proline/), which was used to perform all the data analyses presented. Finally, the authors thanks the anonymous reviewers for their rich and constructive comments.

\section{Author contributions and competing interests}

Y.C. proposed the experimental methods and processed the data. C.B. developed the software environment to perform the experiments and implemented the proposed methodology. T.B. proposed the theoretical framework and drafted the manuscript. All authors contributed to the manuscript and approved its final version. The authors declare no competing interests. 


\section{Supporting Information Available}

- PDF document containing Supporting tools (S1), Supporting results (S2), Supporting methods (S3) and Supporting discussion (S4)

- Zip folder "spectra.zip" containing 150 spectra in PNG format (S5)

\section{References}

(1) Sadygov, R. G.; Cociorva, D.; Yates, J. R. Large-scale database searching using tandem mass spectra: looking up the answer in the back of the book. Nature methods 2004, 1, 195-202.

(2) Cargile, B. J.; Bundy, J. L.; Stephenson, J. L. Potential for false positive identifications from large databases through tandem mass spectrometry. Journal of proteome research 2004, 3, 1082-1085.

(3) Burger, T. Gentle Introduction to the Statistical Foundations of False Discovery Rate in Quantitative Proteomics. Journal of Proteome Research 2018, 17, 12-22.

(4) Elias, J. E.; Gygi, S. P. Target-decoy search strategy for increased confidence in largescale protein identifications by mass spectrometry. Nature methods 2007, 4, 207.

(5) Danilova, Y.; Voronkova, A.; Sulimov, P.; Kertész-Farkas, A. Bias in False Discovery Rate Estimation in Mass-Spectrometry-Based Peptide Identification. Journal of Proteome Research 2019, 18, 2354-2358.

(6) Gupta, N.; Bandeira, N.; Keich, U.; Pevzner, P. A. Target-decoy approach and false discovery rate: when things may go wrong. Journal of the American Society for Mass Spectrometry 2011, 22, 1111-1120. 
(7) He, K.; Fu, Y.; Zeng, W.-F.; Luo, L.; Chi, H.; Liu, C.; Qing, L.-Y.; Sun, R.-X.; He, S.M. A theoretical foundation of the target-decoy search strategy for false discovery rate control in proteomics. arXiv preprint arXiv:1501.00537 2015,

(8) Levitsky, L. I.; Ivanov, M. V.; Lobas, A. A.; Gorshkov, M. V. Unbiased false discovery rate estimation for shotgun proteomics based on the target-decoy approach. Journal of proteome research 2016, 16, 393-397.

(9) Bianco, L.; Mead, J. A.; Bessant, C. Comparison of novel decoy database designs for optimizing protein identification searches using ABRF sPRG2006 standard MS/MS data sets. Journal of proteome research 2009, 8, 1782-1791.

(10) Keich, U.; Tamura, K.; Noble, W. S. An averaging strategy to reduce variability in target-decoy estimates of false discovery rate. Journal of proteome research 2019, 18 , $585-593$.

(11) Jeong, K.; Kim, S.; Bandeira, N. False discovery rates in spectral identification. $B M C$ Bioinformatics 2012, 13, S2.

(12) Käll, L.; Storey, J. D.; MacCoss, M. J.; Noble, W. S. Assigning significance to peptides identified by tandem mass spectrometry using decoy databases. Journal of proteome research 2007, 7, 29-34.

(13) Martínez-Bartolomé, S.; Navarro, P.; Martín-Maroto, F.; López-Ferrer, D.; RamosFernández, A.; Villar, M.; García-Ruiz, J. P.; Vázquez, J. Properties of average score distributions of SEQUEST: the probability ratio method. Molecular $\&$ Cellular Proteomics 2008, 7, 1135-1145.

(14) Cooper, B. The problem with peptide presumption and low Mascot scoring. Journal of proteome research $\mathbf{2 0 1 1}, 10,1432-1435$. 
(15) Cooper, B. The problem with peptide presumption and the downfall of target-decoy false discovery rates. Analytical chemistry 2012, 84, 9663-9667.

(16) Chalkley, R. J. When target-decoy false discovery rate estimations are inaccurate and how to spot instances. Journal of proteome research 2013, 12, 1062-1064.

(17) Bonzon-Kulichenko, E.; Garcia-Marques, F.; Trevisan-Herraz, M.; Vázquez, J. Revisiting peptide identification by high-accuracy mass spectrometry: problems associated with the use of narrow mass precursor windows. Journal of proteome research $\mathbf{2 0 1 4}$, $14,700-710$.

(18) Ezkurdia, I.; Vázquez, J.; Valencia, A.; Tress, M. Analyzing the first drafts of the human proteome. Journal of proteome research 2014, 13, 3854-3855.

(19) Ezkurdia, I.; Calvo, E.; Del Pozo, A.; Vázquez, J.; Valencia, A.; Tress, M. L. The potential clinical impact of the release of two drafts of the human proteome. Expert review of proteomics $\mathbf{2 0 1 5}, \mathbf{1 2}, 579-593$.

(20) Benjamini, Y.; Hochberg, Y. Controlling the false discovery rate: a practical and powerful approach to multiple testing. Journal of the royal statistical society. Series B (Methodological) 1995, 289-300.

(21) Giai Gianetto, Q.; Combes, F.; Ramus, C.; Bruley, C.; Couté, Y.; Burger, T. Calibration plot for proteomics: A graphical tool to visually check the assumptions underlying FDR control in quantitative experiments. Proteomics 2016, 16, 29-32.

(22) Salvetti, A.; Couté, Y.; Epstein, A.; Arata, L.; Kraut, A.; Navratil, V.; Bouvet, P.; Greco, A. Nuclear functions of nucleolin through global proteomics and interactomic approaches. Journal of proteome research 2016, 15, 1659-1669.

(23) Bouyssié, D. et al. Proline: an efficient and user-friendly software suite for large-scale proteomics. Bioinformatics 2020, 36, 3148-3155. 
(24) Efron, B. Large-scale inference: empirical Bayes methods for estimation, testing, and prediction; Cambridge University Press, 2012; Vol. 1.

(25) Klammer, A. A.; Park, C. Y.; Noble, W. S. Statistical calibration of the SEQUEST XCorr function. Journal of proteome research 2009, 8, 2106-2113.

(26) Howbert, J. J.; Noble, W. S. Computing Exact p-values for a Cross-correlation Shotgun Proteomics Score Function. Molecular \& Cellular Proteomics 2014, 13, 2467-2479.

(27) Granholm, V.; Noble, W. S.; Käll, L. On using samples of known protein content to assess the statistical calibration of scores assigned to peptide-spectrum matches in shotgun proteomics. Journal of proteome research 2011, 10, 2671-2678.

(28) Verheggen, K.; Ræder, H.; Berven, F. S.; Martens, L.; Barsnes, H.; Vaudel, M. Anatomy and evolution of database search engines - a central component of mass spectrometry based proteomic workflows. Mass spectrometry reviews 2017,

(29) Bogdanow, B.; Zauber, H.; Selbach, M. Systematic errors in peptide and protein identification and quantification by modified peptides. Molecular \& Cellular Proteomics 2016, 15, 2791-2801.

(30) Barber, R. F.; Candès, E. J., et al. Controlling the false discovery rate via knockoffs. The Annals of Statistics 2015, 43, 2055-2085.

(31) He, K.; Li, M.; Fu, Y.; Gong, F.; Sun, X. A direct approach to false discovery rates by decoy permutations. arXiv preprint arXiv:1804.08222 2018,

(32) Spirin, V.; Shpunt, A.; Seebacher, J.; Gentzel, M.; Shevchenko, A.; Gygi, S.; Sunyaev, S. Assigning spectrum-specific p-values to protein identifications by mass spectrometry. Bioinformatics 2011, 27, 1128-1134.

(33) Colaert, N.; Degroeve, S.; Helsens, K.; Martens, L. Analysis of the resolution limitations of peptide identification algorithms. Journal of proteome research $\mathbf{2 0 1 1}, 10,5555-5561$. 
(34) Vaudel, M.; Burkhart, J. M.; Breiter, D.; Zahedi, R. P.; Sickmann, A.; Martens, L. A complex standard for protein identification, designed by evolution. Journal of proteome research 2012, 11, 5065-5071.

(35) Granholm, V.; Navarro, J. F.; Noble, W. S.; Käll, L. Determining the calibration of confidence estimation procedures for unique peptides in shotgun proteomics. Journal of proteomics 2013, 80, 123-131.

(36) The, M.; MacCoss, M. J.; Noble, W. S.; Käll, L. Fast and Accurate Protein False Discovery Rates on Large-Scale Proteomics Data Sets with Percolator 3.0. Journal of The American Society for Mass Spectrometry 2016, 27, 1719-1727.

(37) The, M.; Edfors, F.; Perez-Riverol, Y.; Payne, S. H.; Hoopmann, M. R.; Palmblad, M.; Forsström, B.; Käll, L. A protein standard that emulates homology for the characterization of protein inference algorithms. Journal of proteome research 2018, 17, 1879-1886.

(38) Storey, J. D. A direct approach to false discovery rates. Journal of the Royal Statistical Society: Series B (Statistical Methodology) 2002, 64, 479-498.

(39) Jagtap, P.; Goslinga, J.; Kooren, J. A.; McGowan, T.; Wroblewski, M. S.; Seymour, S. L.; Griffin, T. J. A two-step database search method improves sensitivity in peptide sequence matches for metaproteomics and proteogenomics studies. Proteomics 2013, 13, 1352-1357.

(40) Huang, T.; Wang, J.; Yu, W.; He, Z. Protein inference: a review. Briefings in bioinformatics 2012, 13, 586-614.

(41) Serang, O.; Noble, W. A review of statistical methods for protein identification using tandem mass spectrometry. Statistics and its interface 2012, 5, 3 .

(42) Alves, G.; Yu, Y.-K. Mass spectrometry-based protein identification with accurate statistical significance assignment. Bioinformatics 2014, 31, 699-706. 
(43) Savitski, M. M.; Wilhelm, M.; Hahne, H.; Kuster, B.; Bantscheff, M. A scalable approach for protein false discovery rate estimation in large proteomic data sets. Molecular 86 Cellular Proteomics 2015, 14, 2394-2404.

(44) Šidák, Z. Rectangular confidence regions for the means of multivariate normal distributions. Journal of the American Statistical Association 1967, 62, 626-633.

(45) Candes, E.; Fan, Y.; Janson, L.; Lv, J. Panning for gold:'model-X'knockoffs for high dimensional controlled variable selection. Journal of the Royal Statistical Society: Series $B$ (Statistical Methodology) 2018, 80, 551-577.

(46) Emery, K.; Hasam, S.; Noble, W. S.; Keich, U. Multiple competition based FDR control. arXiv preprint arXiv:1907.01458 2019,

(47) Lu, Y.; Fan, Y.; Lv, J.; Noble, W. S. DeepPINK: reproducible feature selection in deep neural networks. Advances in Neural Information Processing Systems. 2018; pp $8676-8686$.

(48) Perez-Riverol, Y.; Csordas, A.; Bai, J.; Bernal-Llinares, M.; Hewapathirana, S.; Kundu, D. J.; Inuganti, A.; Griss, J.; Mayer, G.; Eisenacher, M., et al. The PRIDE database and related tools and resources in 2019: improving support for quantification data. Nucleic acids research 2018, 47, D442-D450. 